\title{
Photonic crystal lasers grown on CMOS-compatible on-axis $\operatorname{Si}(001)$
}

\author{
Zhou Taojie ${ }^{1}$, Mingchu Tang ${ }^{2}$, Siming Chen ${ }^{2}$, Huiyun Liu ${ }^{2}$, Zhaoyu Zhang ${ }^{1}$ \\ ${ }^{1}$ School of Science and Engineering, The Chinese University of Hong Kong, Shenzhen, \\ Guangdong, 518172, P.R. China \\ ${ }^{2}$ Department of Electronic and Electrical Engineering, University College London, London, \\ WC1E $7 \mathrm{JE}, \mathrm{UK}$ \\ E-mail: siming.chen@ucl.ac.uk, huiyun.liu@ucl.ac.uk, zhangzy@cuhk.edu.cn
}

\begin{abstract}
Semiconductor photonic crystal (PC) lasers are regarded as promising ultra-compact light sources with ultra-low energy consumption. Here, we demonstrate PC lasers monolithically grown on CMOS-compatible on-axis Si (001) substrate with an ultra-low threshold of $\sim 0.6 \mu \mathrm{W}$. ㅇ 2020 The Author(s)
\end{abstract}

\section{Introduction}

The recent exponential growth in data traffic requires a more efficient on-chip optical interconnection method with lower energy consumption and higher density of processing unit $[1,2]$. In this regard, nanoscale PC cavity with ultra-small mode volume and low energy consumption is one of the most promising architectures for integrated nanoscale devices. Monolithic integration of efficient III-V lightemitting sources on planar on-axis $\mathrm{Si}$ (001) has been recognized as an enabling technology for realizing Si-based photonic integrated circuits (PICs) [3,4]. In addition, zero-dimensional quantum dots (QDs) monolithically grown on Si platform as gain materials provides various advantages, including low lasing threshold, reduced temperature sensitivity, and hence have been widely investigated in last few years.

Here, we present the ultra-small InAs/GaAs QD PC membrane lasers monolithically grown on CMOScompatible Si substrate. The PC laser with a small mode volume of $0.88(\lambda / n)^{3}$ was continuous-wave optically pumped under room temperature, and exhibits an ultra-low lasing threshold of $\sim 0.6 \mu \mathrm{W}$. The demonstrated Si-based PC lasers with a small footprint as well as low power consumption are expected to play an important role in the next-generation nanoscale Si photonics.

\section{Results and Discussion}

The InAs/GaAs QD PC membrane lasers were grown on planar on-axis Si (001) substrates. The active region with 4 layers of InAs/GaAs QDs has been grown between the upper and lower $40 \mathrm{~nm} \mathrm{Al}_{0.4} \mathrm{Ga}_{0.6} \mathrm{As}$ cladding layer, which are grown on the top of $1 \mu \mathrm{m} \mathrm{Al} \mathrm{A}_{0.6} \mathrm{Ga}_{0.4} \mathrm{As}$ sacrificial layer. The QDs within active region present a dot density of $\sim 4 \times 10^{10} \mathrm{~cm}^{-2}$ with a typical size of $25 \mathrm{~nm}$ in diameter and $8 \mathrm{~nm}$ in height. Figure. 1(a) present the schematic diagram of the fabricated InAs/GaAs QD PC laser epitaxially grown on on-axis Si (001) substrate. The top-view (Fig. 1(b)) and tilted cross-section view (Fig. 1(c)) scanning electron microscope (SEM) images of the fabricated PC cavity show smooth etching profiles.
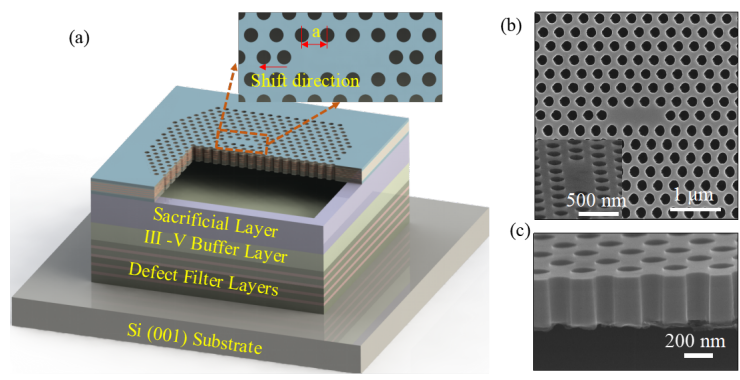

Fig. 1. (a) Schematic diagram of the fabricated InAs/GaAs QD PC (L3 cavity) laser epitaxially grown on on-axis Si (001) substrate. The lattice constant, radius and shift of L3 photonic cavity are a, $\mathrm{r}$ and $0.15 \mathrm{a}$, respectively. (b) Top-view and (c) tilted cross-section view SEM images of the fabricated PC cavity. 
The fabricated PC lasers were continuous-wave (CW) optically pumped at room-temperature using a $632.8 \mathrm{~nm}$ He-Ne laser as the excitation source. The measured spectra under various pumping powers of a single mode $\mathrm{PC}$ laser with $\mathrm{a}=310 \mathrm{~nm}$ and $\mathrm{r} / \mathrm{a}=0.27$ is shown in Fig. 2(a). The collected intensity (L-L) and the linewidth of the lasing peak at $\sim 1306 \mathrm{~nm}$ under various pumping powers are shown in Fig. 2(b), which exhibit the evidence of the lasing with a clear kink of L-L curve and the spectral linewidth narrowing effect. The lasing threshold is estimated to be around $0.6 \mu \mathrm{W}$ from the L-L curve. The inset in Fig. 2(b) shows the calculated Ey field profile of the fundamental mode. In addition, the normalised spectra of various PC lasers above threshold with slightly different radius of air-holes and lattice constant are shown in Fig. 2(c), of which a wide tunable range near $70 \mathrm{~nm}$ is achieved by changing the structural parameters.
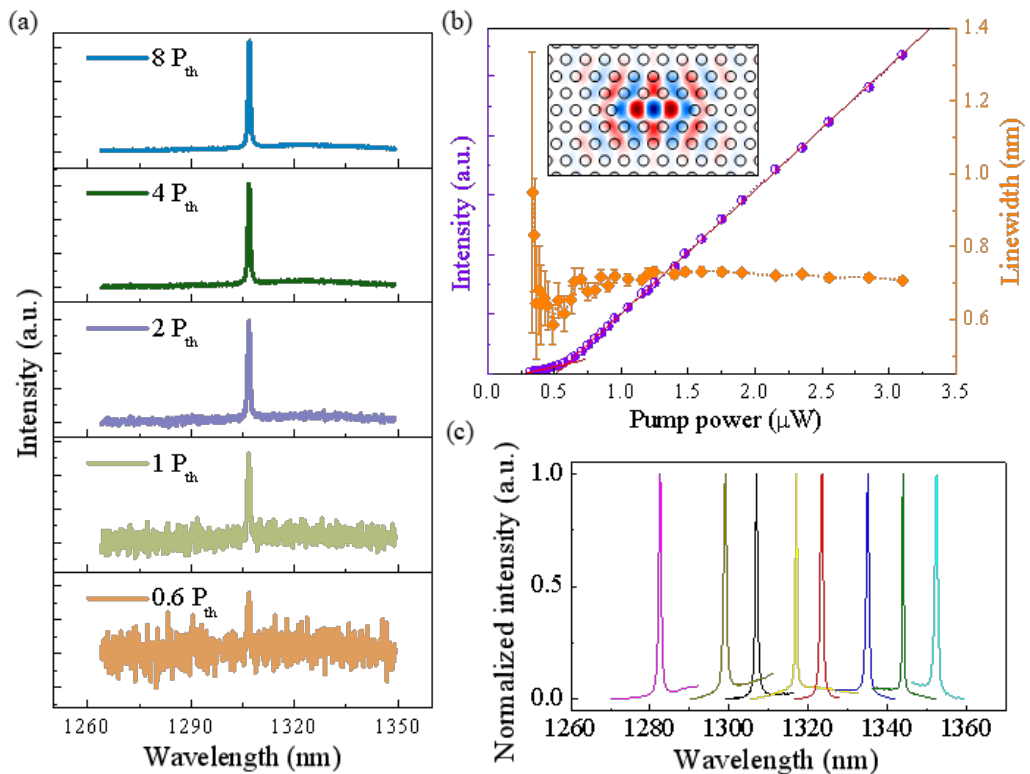

Fig. 2. (a) Measured spectra under various input pumping powers of the PC laser with a $=$ $310 \mathrm{~nm}$ and $\mathrm{r} / \mathrm{a}=0.27$. (b) Collected L-L curve and linewidth of the lasing peak at $1306 \mathrm{~nm}$. The inset shows the calculated $E y$ field profile of the fundamental mode. (c) Normalized PL spectra from representative PC lasers above lasing threshold.

\section{Conclusion}

In conclusion, we report the ultra-small InAs/GaAs QD PC membrane lasers monolithically grown on CMOS-compatible Si substrate. The PC lasers were CW optically pumped at room temperature, exhibiting an ultra-low lasing threshold of $\sim 0.6 \mu \mathrm{W}$. The lasing wavelengths were finely tuned by manipulating the geometrical parameters. The Si-based PC lasers presented in this paper, providing great advantages in terms of a small footprint as well as low power consumption, can be a promising light source in the next-generation nanoscale Si photonics.

\section{References}

1. David AB Miller. Device requirements for optical interconnects to silicon chips. Proceedings of the IEEE, 97(7):1166-1185, 2009.

2. Assaf Shacham, Keren Bergman, and Luca P Carloni. Photonic networks-on-chip for future generations of chip multiprocessors. IEEE Transactions on Computers, 57(9):1246-1260, 2008.

3. Yating Wan, Sen Zhang, Justin C Norman, MJ Kennedy, William He, Songtao Liu, Chao Xiang, Chen Shang, Jian-Jun He, Arthur C Gossard, et al. Tunable quantum dot lasers grown directly on silicon. Optica, 6(11):1394-1400, 2019.

4. Taojie Zhou, Mingchu Tang, Guohong Xiang, Xuan Fang, Xiu Liu, Boyuan Xiang, Suikong Hark, Mickael Martin, Marie-Leonor Touraton, Thierry Baron, et al. Ultra-low threshold InAs/GaAs quantum dot microdisk lasers on planar on-axis Si (001) substrates. Optica, 6(4):430-435, 2019. 